\title{
LA CLASIFICACIÓN DE LA LOCURA EN LA PSIQUIATRÍA ESPAÑOLA DEL SIGLO XIX
}

\author{
José Javier Plumed Domingo
}

Centro Salud Mental Malvarrosa. Valencia

\begin{abstract}
RESUMEN
Los alienistas europeos debatieron intensamente las clasificaciones en psiquiatría durante el siglo XIX. En este artículo revisamos las distintas opiniones sobre nosología publicadas por psiquiatras españoles durante ese período. Los sistemas clasificatorios españoles se estudian en relación con aspectos sociales y teóricos relevantes. Se toman en cuenta las implicaciones reales de esta discusión teórica en la asistencia.
\end{abstract}

PALABRAS CLAVE: Psiquiatría, España, siglo XIX.

\section{SUMMARY}

Classification in psychiatry has been intensely debated all through the nineteenth century by European alienists. In this paper, different nosological views published by Spanish psychiatrists are reviewed. Spanish classificatory systems are studied in relation to social and theoretical aspects relevant to them. Real implications of this theoretical discussion in psychiatric care are taken into account.

KEY WORDS: Psychiatry, Spain, $19^{\text {th }}$ century.

\section{INTRODUCCIÓN}

En el siglo XIX se empieza a constituir el cuerpo de conocimientos de la psiquiatría moderna. El desarrollo de esta disciplina vino acompañado de una explosión nosológica que reflejó las corrientes de pensamiento psiquiátricas que estuvieron en auge en cada período del siglo. Este interés general por la clasificación tuvo como consecuencia una multiplicación de variantes que produjo una sensación de confusión generalizada. Un buen ejemplo de esta situación lo encontramos en un texto de Kalhbaum escrito en su libro sobre la clasificación de las enfermedades mentales, que dice: «el tema de la división de las enfermedades anímicas empieza ya a pertenecer a los objetivos malditos de la investigación personalmente elegida». Haciendo referencia a la discusión noso- 
lógica que se vivió en la psiquiatría alemana del momento. Nombra Kalhbaum en su libro la opinión sobre el tema de algunos de los frenópatas contemporáneos más importantes: «Nasse compara ya en 1818 la incongruencia de las denominaciones en varios autores con la confusión lingüística en la construcción de la torre de Babel». Flemming, al publicar su intento de división (1844), teme provocar, tan sólo con el título «una sensación de desaliento, duda y tedio, como la que de tiempo en tiempo, se origina con el hallazgo de la cuadratura del círculo por un matemático». Baillarger, al postular una nueva forma de trastorno anímico y justo después de haber tratado el tema de la clasificación en general, se disculpa señalando: «no ignoro que, bien a menudo se repite y no sin razón que las distinciones nuevas, que tienden sin cesar a modificar las clasificaciones, son más a menudo nocivas que útiles al progreso de la ciencia» ${ }^{1}$.

Vamos a ver las líneas más significativas de evolución en la nosología psiquiátrica del período. En un momento inicial, Pinel y Esquirol establecieron un modelo nosográfico basado en la clínica. Especialmente en el caso de Esquirol, se evidencia un gran esfuerzo para diferenciar cuadros psiquiátricos basados en exhaustivas descripciones que permitiesen diferenciar modos distintos dentro del cuerpo común de la locura. Lanteri-Laura ${ }^{2}$ considera que en este primer período hay una definición de formas de manifestación de una enfermedad única. Sin embargo, la diferenciación de la locura en especies, de acuerdo con Pinel, o los géneros descritos por Esquirol marcan la necesidad de hacer una distinción dentro de la unidad general con vistas al tratamiento y estudio de la alienación. En un párrafo de su libro, Tratado de la manía, Pinel ${ }^{3}$ describió la importancia que daba a la idea de establecer distintas especies morbosas. Por su parte, Esquirol señaló en su Tratado el «error de los prácticos que sólo han visto una enfermedad en todas las locuras que han combatido» ${ }^{4}$.

1 Kalhbaum, L. (1863), Die Gruppirung des psychischen Krankheinten und die Eintheilung der Seelenstötungen, Danzig. El texto consultado es una traducción de 1995 titulada Clasificación de las enfermedades mentales, Madrid, DOR, p. 36.

2 LANTERI-LAura, G. (1998), Essais sur les paradigmes de la psychiatrie moderne, París, Du Temps. La edición consultada es una traducción titulada Ensayo sobre los paradigmas de la psiquiatría moderna, Madrid, Triacastela, pp. 80-87.

3 Pinel, P. (1804), Tratado médico-filosófico de la enagenación del alma o manía, Madrid, Imprenta Real, p. 74. Traducción del original de 1800, Traité médico-philosophique sur l'alienation mentale, París, Caille et Ranvier.

4 EsQuirol, J. E. D. (1838), Des maladies mentales considérées sous les rapports médical, hygiénique et médico-legal, Paris, Chez-Baillière. El texto consultado proviene de la traducción española de 1856, Tratado completo de las enagenaciones mentales consideradas bajo su aspecto médico, higiénico y médico-legal, Madrid, Imp. de Gómez Fuentenebro, p. 121. 
De hecho, esta ambivalencia entre los modelos nosográficos y antinosográficos ha sido señalada por diversos autores ${ }^{5}$.

Pinel ha sido considerado un autor psicologicista, en el sentido de que consideraba la enfermedad mental como enfermedad del alma, no como la consecuencia de una disfunción orgánica del cerebro. Los autores psicologicistas eran dualistas y consideraban los conceptos de mente y alma como virtualmente idénticos. Esto daba lugar a importantes problemas. Por un lado, el pensamiento cristiano tradicional no podía admitir una perturbación del alma. Por eso, sólo podía considerar la locura como una alteración del sustrato material del alma, el cerebro, mediante un mecanismo no bien conocido. De la misma forma, el funcionamiento espiritual podía afectar al cerebro ${ }^{6}$. Además, los tratamientos debían estar basados en los medios morales, ya que sólo estos afectaban a la esencia del proceso morboso de la locura. Los autores espiritualistas perdieron peso en la segunda parte del siglo, a medida que el cientifismo fue cobrando interés entre los frenópatas ${ }^{7}$.

Un hito dentro de la nosología psiquiátrica fue la obra de Bayle. Con su trabajo sobre la parálisis general, estableció por primera vez en la historia de la psiquiatría una verdadera entidad nosológica, de acuerdo con el modelo anatomoclínico vigente en el momento. Además del descubrimiento de una lesión específica, la parálisis general mostraba un modelo de enfermedad única con manifestaciones clínicas diferentes en el tiempo ${ }^{8}$ y sirvió para extender la creencia en la eficacia de una metodología de trabajo, el estudio anatomopatológico del cadáver. Con esto, los modelos tradicionales, basados en la enfermedad mental entendida como una variación cuantitativa respecto a la

5 Huertas, R. (1999), « Nosología y antinosología en la Psiquiatría del siglo XIX: en torno a la psicosis única», Revista de la Asociación Española de Neuropsiquiatría, 19, 69, pp. 63-76.

6 Wallace, E. (1994), Psychiatry and its Nosology. En: Sadler, J.; Wiggins, O.; Schwartz, M., Philosophical Perspectives on Psychiatric Diagnostic Classification. Nueva York, Johns Hopkins, p. 32.

7 Autores como Eric Hobsbawn señalan la admiración de la sociedad de mediados del siglo XIX hacia el progreso científico y su tendencia a subordinarle cualquier forma de desarrollo intelectual. Reproduce este autor en su libro un texto de 1861 del economista francés Cournot que observaba que «la creencia en la verdad filosófica se ha enfriado tanto que ya ni el público ni las academias gustan de recibir o dar la bienvenida a obras de esta clase, excepto como producto de la erudición pura o como curiosidad histórica». En: HoBSBAwn, E. (1998), La era del capital 1848-1875, Barcelona, Crítica, p. 263.

8 BERCHERIE, P. (1980), Les fondements de la clinique. Histoire et structure du savoir psychiatrique, París, Navarin. La edición manejada es la traducción al castellano titulada Los fundamentos de la clínica. Buenos Aires, Manantial, p. 54. 
normalidad se sustituyeron por las teorías ontológicas que consideraban la locura como un grupo diferenciado de enfermedades con distinta lesión anatómica ${ }^{9}$. De forma especulativa, aparecieron clasificaciones supuestamente basadas en lesiones anatómicas o funcionales que pretendían haber alcanzado el nivel de desarrollo del resto de las especialidades médicas. Sin embargo, las críticas abundaron, ya que las salas de autopsias no daban las respuestas que muchos esperaban encontrar.

Las cuestiones sobre la etiología y patogenia de la locura encontraron varias propuestas. El psiquiatra más destacado de la escuela alemana, Griesinger, defendió un modelo antinosográfico, que partía de que la locura es un proceso único de base afectiva. Así, en un momento inicial aparecen los cuadros afectivos - manía y melancolía -; posteriormente los trastornos de la percepción y la voluntad y, si avanza el proceso, la demencia. Este modelo estaba inspirado en la obra del frenópata belga Guislain, que propuso que el sufrimiento melancólico o «frenalgia» era la base de toda enfermedad mental. Si se daban los factores causales suficientes, dicha frenalgia podía transformarse a la manía, parafrenia, epilepsia, delirio y demencia ${ }^{10}$.

Por otro lado, el modelo etiológico, que triunfó en medicina con la teoría infecciosa de Pasteur, fue asumido en psiquiatría por la teoría de la degeneración. Proponía que la enfermedad mental estaba producida por un proceso fundamental, la degeneración. Se debía a la herencia enfermiza de los antecesores y, a su vez, determinados factores tóxicos como el alcoholismo podían agravar o estimular el proceso. Estaba fuertemente condicionada por las teorías evolucionistas de Lamark y por factores ideológicos de tipo religioso. Las distintas enfermedades se producían de acuerdo con las fases del proceso degenerativo. En las sucesivas generaciones de afectados aparecían las distintas enfermedades mentales con el siguiente orden: en la primera generación se daba el predominio de lo que llamó «temperamento nervioso», y que se manifestaba por determinados síntomas como: irritabilidad, violencia y alteraciones de carácter; en la generación sucesiva se producían los cuadros epilépticos, histéricos e hipocondríacos; y en la generación siguiente la locura propiamente dicha ${ }^{11}$. Su discípulo, Valentín Magnan, adoptó un punto de vis-

9 Boyle, M. (1990), Schizophrenia. A Scientific Delusion?, Londres, Routledge, pp. 39-41.

10 Berrios, G.E.; Beer, D. (1995), Unitary Psychoses Concept. En: Berrios, G.; PorTER, R., History of Clinical Psychiatry, Londres, Athlone Press, pp. 321-323.

11 Esta idea del pensamiento de Morel aparece en su libro MoRel, B. (1860), Traité des maladies mentales, París, Masson, pp. 515-516. 
ta aconfesional e incidió en aspectos claves para la nosología ${ }^{12}$. Entre ellos, destacó su interés por prestar una especial atención al curso y pronóstico de la enfermedad mental. De hecho, la teoría de la degeneración fue una de las más influyentes en la psiquiatría europea de la segunda parte del siglo XIX.

La obra de Kalhbaum inició un momento clave en la clasificación psiquiátrica. A diferencia de los criterios especulativos de otros autores, intentó establecer una clasificación basada en el estudio del comienzo de los cuadros, su curso y el desarrollo final. Tomó como lema las palabras: «deseo observar la naturaleza, pero ser cuidadoso de no pensar» ${ }^{13}$. A partir de este principio definió la catatonia, como una entidad nosológica descrita gracias a la observación clínica, sin ningún presupuesto teórico subyacente. Posteriormente, Kraepelin estuvo marcado por esta actitud «empíricoclínica» a la hora de establecer la distinción entre la demencia precoz y la psicosis maníaco-depresiva ${ }^{14}$.

En este trabajo, vamos a estudiar las peculiaridades que la clasificación psiquiátrica tuvo en la psiquiatría española.

\section{LA NOSOLOGÍA INICIAL DE LA ESCUELA ESPAÑOLA}

Los psiquiatras españoles explotaron los modelos nosológicos provenientes de las escuelas europeas, especialmente la francesa. Así, nos encontramos en la primera parte del siglo con una asunción acrítica del modelo de Pinel y, posteriormente, Esquirol. Un ejemplo de esta tendencia la encontramos en un artículo de Ballarin, donde comenta una clasificación de la enfermedad mental tomada de los modelos de Esquirol y Pinel. Respecto a la naturaleza de la clasificación opina: «todos los grupos de síntomas por los que se denominan las especies anteriores no constituyen más que diferentes grados de una misma enfermedad»; «la locura es el máximum o extremo de una pasión o de una afección; así lo cree el célebre Esquirol» ${ }^{15}$. Ya hemos comentado en la introducción que Esquirol y Pinel tuvieron una ambivalencia constante entre el

12 Sobre los conceptos ligados a la teoría de la degeneración ver: HuERTAS, R. (1992), «Madness and degeneration. From «fallen angel» to mentally ill», History of Psychiatry, 3, pp. 391-411.

13 La frase está tomada de: ZILboorg, G. (1941) A History of Medical Psychology, Nueva York, Norton, p. 448.

14 Berrios, G.E., Hauser, R. (1988), «The early Development of Kraepelin's ideas on Classification: a Conceptual History», Psychological Medicine, 18, pp. 813-821.

15 BALlarin, F. (1835), «Memoria sobre el establecimiento de dementes de Zaragoza», Gaceta Médica de Madrid, 1, pp. 367-70; 374-8; 386-8. 
modelo nosológico y antinosológico, que asumieron los autores que introdujeron sus conceptos en nuestro país.

Un autor tan significativo como Rodríguez Villargoitia ${ }^{16}$ señala, en 1847, el problema de lo limitado de las clasificaciones del momento para incluir toda la variedad sintomática de la enfermedad mental: «la nosología mental de los más célebres nosógrafos no basta para colocar todas las variedades que se observan en los establecimientos»; ¿a qué lugar corresponde en las clasificaciones a los ebrios, orgullosos, fanáticos, avaros y otros que frecuentemente se ven abandonados a la razón y se entregan a trascendentes desvaríos?» ${ }^{17}$. Sin embargo, en el artículo menciona los grandes grupos propuestos por Pinel y Esquirol, tales como: demencia, manía, alucinación e ilusión, sin que aparezca una propuesta nosológica diferente

Esta defensa de los planteamientos tradicionales de la psiquiatría francesa continúa en distintos autores hasta la segunda parte del siglo. Podemos encontrarla en el planteamiento nosológico de la ponencia de Quintana titulada « $\mathrm{Pa}-$ sión y locura» ${ }^{18}$, donde se define la enfermedad mental de acuerdo con el principio clásico que Esquirol toma de la medicina ilustrada: la locura supone una pérdida de la razón por la imposibilidad de controlar las pasiones. Dos facultades psíquicas: la reflexión y la voluntad, se convierten para el autor en las facultades psicológicas que caracterizan el pensamiento racional. La salud mental está condicionada para Quintana por la medida en que estas dos facultades psíquicas están conservadas. El peso que este autor concede a la voluntad le hace partícipe de una corriente de frenópatas para los que esta facultad era central a la hora de entender la vida psíquica. Junto a los aspectos teóricos y clínicos, existieron factores profesionales e ideológicos que justifican el interés por los aspectos volitivos de la conducta por parte de la psiquiatría en este momento histórico. El primero, extensamente estudiado en la historiografía ${ }^{19}$, se basaba en el problema de la intervención forense del alienista, en cuanto a que un cri-

16 Rodríguez Villargoitia fue médico titular de la sala de dementes del Hospital General de Madrid y uno de los frenópatas españoles más significativos de la primera mitad del siglo XIX. Sobre el autor destaca el trabajo: REY, A (1984), «Clásicos de la medicina española del siglo XIX: José Rodríguez Villargoita», Revista de la Asociación Española de Neuropsiquiatría, 4/10, pp. 264-275.

17 Villargoitia, J. R. (1847), «Consideraciones sobre las enfermedades mentales», La Facultad, 2, 137-138; 151-154; 167-170; 186-187; 214-215, p. 214.

18 QuintanA, J. (1863), «Discurso pronunciado sobre la «pasión y la locura» en la Real Academia de Medicina de Madrid», Siglo Médico, 10, 341-4; 357-9; 373-5; 390-2.

19 Sobre este tema destaca el estudio de: GoldsteIn, J (2001), Console and Classify: the French Psychiatric Profession in the nineteenth Century, Londres, Chicago Press, pp. 166-169. 
men sólo se consideraba como tal cuando al individuo se le suponía dueño de sus actos. El alienista afirmaba poseer los conocimientos profesionales necesarios para determinar objetivamente si el reo era responsable o no. En segundo lugar, la religión católica tenía un gran peso social en España y su influencia se dejaba ver en el debate científico. El libre albedrío y su afectación o no en la enfermedad psiquiátrica era una cuestión determinante, ya que la idea de pecado se sostenía en función de la capacidad de elegir del individuo ${ }^{20}$.

Vemos cómo el modelo de clasificación de Quintana, aunque parte de la posibilidad de distinguir formas distintas, defiende que la locura es, en su esencia, un trastorno único, como la mayoría de frenópatas españoles de la primera mitad del siglo. Se relaciona desde lo psicológico con el nuevo pensamiento fisiopatológico: la locura es un proceso dimensional y el grado de alteración de las variables psicológicas esenciales condiciona sus diferentes manifestaciones. Ya desde las aportaciones de Claude Bernard a la medicina, se considera que los procesos orgánicos funcionales se desarrollan de forma continua, lo que encuentra una similitud en el campo de la psiquiatría con la hipótesis basada en el efecto patogénico gradual de las pasiones. Así encuentra su justificación etiopatogénica la nosología de Esquirol.

Muchos otros autores expusieron sus propias clasificaciones de orientación psicologicista, sin que aportasen nada relevante respecto a las ideas de Pinel y Esquirol. Por ejemplo, Aguilar y Calpe ${ }^{21}$ defiende como propia una clasificación que divide a la locura en dos tipos. En el primer grupo se afectan los sentimientos y los actos, lo que hace que la locura sea afectiva. El segundo grupo incluye a los trastornos mentales solo afectados en el orden de las ideas, lo que hace que la locura sea intelectual o racional. A este segundo grupo lo engloba bajo el término manía. Dentro de este grupo, si predomina la exaltación tendremos una monomanía y una melancolía en el caso de que exista depresión. En cualquiera de estas formas vesánicas la demencia puede producirse y se trata de una complicación desgraciada en el curso de la enfermedad y no parte de la evolución normal del proceso.

Al contrario de lo que veíamos en Quintana, Aguilar y Calpe no considera que la voluntad sea una facultad superior, responsable última de las acciones humanas. Para él, los trastornos de esta facultad están relacionados con los

20 Sobre el debate acerca del libre albedrío en el alienismo español, ver: Diéguez, A. (2002) El problema del libre albedrío en el alienismo español; En: FuenteneBro, F.; HuerTAS, R.; VAliEnTE, C., Historia de la psiquiatría en Europa, Madrid, Frenia, pp. 137-146.

21 Aguilar y CALPe (1880), «La Civilización no es culpable de la locura», Boletín del Instituto Médico Valenciano, 16, 335-356. 
afectos y carecen de autonomía. Un tema como este, el carácter primario o secundario de la voluntad humana, justificaba intensos debates en ámbitos académicos ${ }^{22}$.

\section{LA CLASIFICACIÓN DE LOS AUTORES ESPIRITUALISTAS}

Uno de los mejores ejemplos de esta orientación teórica en la clasificación es el trabajo clásico de Crous y Casellas ${ }^{23}$, catedrático de Patología General en la Universidad de Valencia. Este autor propugna aquí como fundamento teórico para la clasificación frenopática un «somaticismo espiritualista», que sería una forma de eclecticismo entre las posturas más radicales sostenidas por autores materialistas y espiritualistas ${ }^{24}$.

Si buena parte de la discusión teórica en las décadas previas se había basado en cuestiones de naturaleza filosófica, en la segunda mitad del siglo XIX había un desprecio generalizado a todo análisis especulativo. La bandera ideológica del nuevo siglo la constituían los nuevos avances científicos y todos los intentos racionalistas se consideraban arcaicos. Esto hacía que todo intento de clasificación psiquiátrica para ser veraz tuviera que estar apoyado por datos aparentemente empíricos y objetivos, como es el caso de la clasificación de Crous y Casellas.

Los autores tradicionales y conservadores defendían el dogma de la iglesia católica y consideraban peligroso y nocivo cualquier intento de cuestionarlo. Uno de los asuntos más discutidos era la dualidad alma-cuerpo y cómo sostener esta dicotomía en el estudio de las perturbaciones psíquicas. Por ello, los intentos laicos de reducción al materialismo eran rechazados por estos autores $^{25}$. Crous y Casellas intenta en su ensayo armonizar estos dos aspectos. Vamos a ver con más detalle el fundamento de su clasificación.

22 Berrios define como reduccionistas a aquellos autores que supeditan la voluntad a las otras funciones del psiquismo humano y no reduccionistas a aquellos que la consideran independiente. Esto generó un importante debate histórico. Ver: BERRIOS, G.E.; GILl, M. (1995), «Will and its Disorders: a Conceptual History», History of Psychiatry, 6, 87-104.

23 Crous y CASEllas, J (1881), « Fisiología patológica de las enfermedades mentales», Boletín del Instituto Médico Valenciano, 17, 346-63; 373-84.

24 Acerca de este trabajo, recordamos el trabajo de Peset Llorca, PESET, V. (1962), «Los «Elementos de Frenopatología» de Crous y Casellas, con algunos comentarios», Cuadernos de Historia de la Medicina Española, I, 195-212.

25 Autores materialistas, como los alemanes, no fueron muy traducidos en España hasta el último tercio del siglo XIX. Uno de los filósofos más representativos de esta escuela fue 
Comienza su trabajo criticando los dos fundamentos teóricos que sustentan las clasificaciones frenopáticas. Por un lado, la corriente positivista, que piensa que reduce el análisis de la experiencia mental a su correlación con el sustrato anatómico sin recordar que «la enajenación es las más veces un padecimiento dinámico». Por otro lado está la corriente espiritualista, que defiende que los padecimientos frenopáticos están producidos por una alteración en la parte volitiva, intelectiva o afectiva del alma. Crous y Casellas considera reduccionista este punto de vista, ya que «hasta los filósofos griegos» reconocen en las manifestaciones psíquicas una influencia del sustrato orgánico. Su postura la define como un estado intermedio entre los anteriores, que define como somaticismo espiritualista y que fundamenta en la filosofía de Santo Tomás de Aquino. No es extraño el que Crous y Casellas escoja este fundamento teórico, ya que la orientación tomista en la psicología y psiquiatría españolas de la segunda parte del siglo fue una constante entre los autores de orientación espiritualista. Es importante recordar en este sentido que el Papa León XIII en una encíclica de 1879 estimulaba la vuelta de los filósofos cristianos al pensamiento de Santo Tomás, como la réplica cristiana a las nuevas cuestiones propuestas por la ciencia frente al evolucionismo y la ciencia materialista alemana ${ }^{26}$. El tomismo propone un fundamento filosófico desde el catolicismo para entender el equilibrio mutuo entre el alma y el cuerpo y sus interacciones, que subyace el pensamiento de frenópatas católicos como $\mathrm{Morel}^{27}$.

El vitalismo, la doctrina más importante de la medicina del siglo XVIII ${ }^{28}$, fue el modelo al que los autores espiritualistas recurrieron para rechazar el

Ludwig Bückner. En sus conferencias sobre las relaciones de la teoría darwinista con la doctrina del progreso y la filosofía materialista, señala como uno de los principios irrefutables representado por las conquistas de la ciencia la certeza fisiológica de que el cerebro es el órgano del alma y el abandono en las ciencias de la naturaleza del principio vital, de la idea de las causas finales y en suma, de todas las fuerzas místicas. Ver: PELAyo, F.(1999), Ciencia y creencia en España durante el siglo XIX, Madrid, CSIC, pp. 276-277.

26 Ver: CARPINTERO, H. (1994), Historia de la psicología en España, Madrid, EudemA, p. 108.

27 Sobre la influencia de la filosofía de Tomás de Aquino a la hora de estudiar la interacción alma-cuerpo ver: BERRIOS, G.E. (2000), La etiología en psiquiatría. En: LuQue, R.; VILlagrán, J., Psicopatología descriptiva: nuevas tendencias, Madrid, Trotta, pp. 561-562.

28 El vitalismo fue el resultado de la búsqueda de una vía intermedia entre el mecanicismo y el animismo para explicar los fenómenos psicológicos y patológicos y, en último extremo, la consistencia de la vida. Rechazó la idea de seres vivos reducidos a complicadas máquinas físicas o químicas, así como el recurso al ánima o cualquier otro factor ajeno al organismo, e intentó dar razón de la peculiaridad de la vida, tanto en estado de salud como de enfermedad, por medio de una fuerza o cualidad intrínseca de la materia orgánica. Fue base de 
reduccionismo materialista que veían en los modelos anatomopatológico y fisiopatológico de la enfermedad mental. No fue una excepción Crous y Casellas y recurre a esta doctrina en su estudio. El principio de vida que anima el organismo humano y que tiene facultades nutritivas, motoras, sensitivas e intelectivas es, para este autor, la esencia de la naturaleza humana. De acuerdo con el tomismo, hay una interación continua entre el cuerpo y este principio vital, cuyo mecanismo debe estudiarse desde la ciencia. Así, el reflejismo es para Crous y Casellas el mecanismo fisiopatológico por excelencia que justifica científicamente esta interacción. Por su parte, las trasgresiones morales pueden actuar sobre la materia orgánica mediante otros mecanismos, aunque este autor carece de un modelo teórico que los explique.

En su clasificación, este autor cae en la especulación teórica para justificar su eclecticismo y llega a establecer correlaciones directas entre lesiones cerebrales y enfermedades psiquiátricas. Así, postula que las ilusiones y alucinaciones se producen por afectación de los elementos sensoriales del nervio óptico; las locuras afectivas se deben a la afectación de la capa más superficial del cerebro y las locuras impulsivas se producen por la afectación de las células más profundas de la corteza cerebral. En las monomanías el cambio producido por el delirio se limita a una pequeña esfera, tanto a nivel psíquico como a nivel de la extensión del daño en el cerebro. Por otro lado, en las locuras generalizadas como la manía y la demencia, el daño reviste una amplitud explicable por la afectación de todas las capas del cerebro.

La orientación espiritualista para el estudio de la locura cayó en franca decadencia a partir de la segunda parte del siglo, en relación con el espectacular avance del somaticismo, como veremos en el siguiente punto del trabajo.

Fig. 1. ClasifiCACiÓN DE CROUS Y CASELLAS.

1. Locura perceptiva.

2. Locuras intelectuales.

2.1. Monomanía intelectual.

2.2. Manía intelectual crónica.

2.3. Manía razonadora

2.4. Impulsos morbosos intelectuales subjetivos.

2.5. Impulsos morbosos intelectuales objetivos.

la ideología médica de la Ilustración. En: LóPEZ PIÑERo, J.M. (2002), La Medicina en la Historia, Madrid, La esfera de los libros, p. 352. 
3. Locuras afectivas.

3.1. Monomanía afectiva.

3.2. Melancolía simple.

3.3. Melancolía con delirio.

3.4. Melancolía con estupor.

3.5. Manía hipocondríaca e histérica.

3.6. Locura epidémica.

4. Locuras volitivas.

4.1. Impulsos morbosos volitivos.

4.2. Abulomanias.

5. Locuras compuestas.

5.1. Locura periódica.

5.2. Hebefrenia.

5.3. Locura circular.

5.4. Catatonia.

5.5. Demencia primitiva

6. Locuras constitucionales.

6.1. Locura epiléptica.

6.2. Locura puerperal.

6.3. Locura pelagrosa.

6.4. Locura coreica.

7. Suspensión del desarrollo mental.

7.1. Idiotismo.

7.2. Cretinismo

\section{LA ESCUELA CATALANA. El TRIUNFO DEL ORGANICISMO}

Aunque no todos los autores comparten la idea de que en la España del siglo XIX existieron escuelas psiquiátricas, sino más bien núcleos de actividad $^{29}$, es bien conocido que el grupo de frenópatas catalanes fue uno de los más pujantes y prestigiosos en ese momento histórico.

29 Rafael Huertas comenta la dificultad de hablar en el siglo XIX de «escuelas psiquiátricas» propiamente dichas. Para este autor, el escaso grado de institucionalización y los esfuer- 
En aquel tiempo las clasificaciones de la locura se basaron en la población manicomial. De esta forma, como en otros países, se excluyeron aquellos trastornos que quedaron incluidos en el grupo de las neurosis y se estudiaron las manifestaciones psíquicas que presentaban los pacientes ingresados en los asilos. Así, hay una estrecha relación entre la estructura de la asistencia manicomial y la nosología psiquiátrica. En Cataluña se fundaron las instituciones psiquiátricas privadas más importantes. La primera fue el Sanatorio de San Baudilio de Llobregat, dirigido por Antonio Pujadas y Mayans, que fue estéril a nivel de producción científica y del que finalmente se hizo cargo Arturo Galcerán Granés ${ }^{30}$. Pero la institución privada más relevante fue el Manicomio Nueva Belén, dirigido por la que, quizás, sea la figura más prestigiosa del alienismo español del siglo XIX, Juan Giné y Partagás ${ }^{31}$.

El ingreso en el manicomio debía estar justificado por el carácter científico y especializado de la institución. Por ello, el modelo de la enfermedad mental tenía que acercarse a la ciencia moderna, especializada y eficaz, que se propagaba en los países europeos y diferenciarse del paupérrimo panorama asistencial de los sanatorios públicos.

Giné y Partagás ${ }^{32}$ defiende un modelo médico de enfermedad mental que no sea distinto del de otras especialidades médicas: «decir que para los estudios frenopáticos es indispensable el conocimiento de las funciones de los centros nerviosos, equivale a enunciar una proposición que, si de algo peca, es de trivial ${ }^{33}$. En este marco ideológico se asienta una psiquiatría que requiere de especialistas formados como en cualquier otra disciplina médica y de manicomios donde se introduzcan los adelantos técnicos requeridos para un diagnóstico y tratamiento adecuados.

Sin embargo, no resultaba ajeno a Giné y Partagás el hecho de que todavía no aparecían lesiones materiales en la gran mayoría de las enfermedades men-

\footnotetext{
zos legitimadores, no siempre exitosos, de los primeros alienistas hace que se pueda hablar de «núcleo de actividad» antes que de escuelas. Ver: HuERTAS, R. (2002), Organizar y persuadir, Madrid, Frenia, p. 75.

30 Ver: EsPINOSA, J. (1966), La asistencia psiquiátrica en la España del Siglo XIX, Valencia, Cátedra e Instituto de Historia de la Medicina, pp. 110-113.

31 Sobre este autor, ver: REY, A. (1975), «Joan Giné y Partagás», Revista de la Asociación Española de Neuropsiquiatría, 2 (4), 231-234.

32 Un buen estudio sobre la nosología en la obra de Giné y Partagás aparece en: DIÉGUEZ, A. (1998), «El problema de la nosografía en la obra de Giné y Partagás», Asclepio, 50 (1), 199-221.

33 GinÉ y PARTAgÁs, J. (1876), Tratado teórico-práctico de frenopatología, Madrid, Imp. Moya y Plaza, p. 68.
} 
tales ${ }^{34}$, por lo que la nosología no podía construirse de acuerdo con el modelo anatomoclínico de enfermedad. De hecho, establece una clasificación de enfermedades con un modelo patogénico basado en un trastorno emocional básico, un desorden funcional como en los modelos de Griesinger y Guislain. Así, la manía supone una exaltación de la afectividad y la inteligencia, sin delirio o con delirio general; la melancolía un predominio de los sentimientos tristes; la monomanía una «exaltación de los sentimientos expansivos» y el éxtasis: «suspensión de los actos intelectuales y estéticos con rigidez general». Por último están las locuras patogenéticas, «sostenidas por agentes tóxicos». Todas estas formas son susceptibles de tratamiento. Sin embargo esto no sucede con el grupo de las demencias, ya sean de tipo primario o secundario a otra enfermedad mental, así como con los defectos frénicos como el idiotismo y la imbecilidad.

La obra de Guislain y sus «Lecciones orales sobre las enfermedades mentales» aparecen constantemente citadas en su Tratado. La obra de Guislain es conocido que se trata de uno de los intentos más importantes de la psiquiatría del siglo pasado de sostener la hipótesis de la psicosis única. Giné y Partagás, en el libro, oscila entre este modelo antinosológico y un acercamiento nosológico a la clasificación. Por un lado, el fondo radical afectivo inicial propuesto por Guislain parece aceptarlo Giné en buena parte de su libro. En este sentido, define la patogenia de la enfermedad mental de la siguiente forma: «del mismo modo que el dolor constituye la primera expresión de la enfermedad somática, la pena, la ansiedad, la tristeza o el mal humor suelen ser las manifestaciones iniciales del estado frenopático». Sin embargo, la aproximación racional de Guislain para interpretar la clínica resulta insuficiente para Giné, que necesita justificar desde el punto de vista anatómico y fisiológico este proceso: «la causa inmediata de las perturbaciones psíquicas reside en las células afectivas de la capa cortical de los hemisferios»; "se ignora a punto fijo cuáles sean estas células, por más que haya motivos para suponer que están interpoladas e íntimamente enlazadas con las intelectuales. Poseidas de eretismo nervioso, las células afectivas dominan a las intelectuales, las cuales a su vez participan de la conmoción que experimentan aquellas, para no percibir ni juzgar de manera anómala, esto es, según la forma del estado emocional dominante» ${ }^{35}$. Así, la observación clínica no se convierte en una base suficiente para la clasificación, sino que ha de haber un sofisticado aparato teórico basado en una fisiología y anatomía cerebrales definida. La búsqueda

34 Giné Y PARTAGÁs, J. (1876), p. 238.

35 GINÉ Y PARTAGÁs, J. (1876), p. 128-129. 
de lesiones anatómicas diferenciadas y trastornos funcionales específicos parece más cercana a una actitud nosológica y encuentra su referente en autores alemanes como Meynert. En relación con esto hay una diferenciación de géneros y especies de acuerdo con el modelo anatomo y fisiopatológico de enfermedad. Esto hace que la parálisis general sea considerada como un género distinto y la locura de doble forma una simple complicación de la manía, en cuanto el criterio evolutivo no ha madurado en Giné y busca la afectación material subyacente como base nosológica.

De hecho, los frenópatas catalanes se sentían así más seguros de la calidad de su ciencia, en la medida que habían llegado a una frenopatología de orientación positivista y materialista.

A pesar de que parece adoptar un modelo científico y aconfesional, Giné y Partagás mantiene la idea tradicional de que el alma preside en último lugar el comportamiento humano al menos, quizás, como consecuencia de la necesidad de no crear conflictos ante una sociedad demasiado conservadora ${ }^{36}$. Cuando habla en su Tratado sobre la voluntad, opina: «al pasar al terreno donde se dan los movimientos voluntarios, encontramos un punto donde no es posible no aceptar la actividad directa del alma y ese punto es la voluntad»». Decir que la voluntad está subordinada a la sensibilidad y a la inteligencia es enunciar una verdad; pero no es explicar ni la esencia ni el origen de esa fuerza, que en nada se parece a las que animan la materia» ${ }^{37}$.

Para Giné y Partagás la finalidad última de una clasificación es «proporcionar el mayor número posible de utilidades prácticas ${ }^{38}$. Es decir, el diagnóstico correcto permite un tratamiento eficaz y la institución manicomial ha de clasificar a los enfermos en función de su patología, de modo que sean complementarias: «la clasificación se funda en las condiciones de la enfermedad. En este punto es preciso abandonar toda idea especulativa y atenerse a las necesidades de la práctica. Fácil es comprender los inconvenientes que resultarían para los enfermos si se hallasen constantemente reunidos los afectados de una misma categoría de enfermedades mentales», «el triste y taciturno, al lado del hablador tranquilo, y este junto al monomaníaco filosofante, equilibran, por el recíproco trato, sus individuales disposiciones frenopáticas $\rangle^{39}$. Sin embargo, las indica-

36 En un momento tan tardío como 1868, se exigía en España una censura previa eclesiástica para aquellas publicaciones que pudieran tener relación con el dogma católico. Ver: Pelayo, F (1999), p. 191.

37 Giné y PARTAGÁs, J. (1876), p. 95-96.

38 GINÉ Y PARTAGÁs, J. (1876), p. 238.

39 Giné y PARTAGÁs, J. (1876), p. 323-324. 
ciones del Tratado de Giné sobre la organización de los enfermos hace referencia antes a la manifestación conductual más grosera que presente el paciente que a cualquier sofisticación nosológica. De hecho, en otro trabajo comentamos cómo los casos de Nueva Belen ${ }^{40}$ se trataban atendiendo al síndrome clínico aparente y no a la etiqueta diagnóstica.

\section{Fig. 2. ClasificACIÓN DE GinÉ Y PARTAGÁS.}

1. Orden: Locuras.

1.1. Géneros: manía, melancolía, monomanía, éxtasis, locuras patogenéticas.

2. Orden: Demencias

2.1. Géneros: parálisis general, demencias propiamente dichas.

3. Orden: Defectos de desarrollo frénico.

3.1. Géneros: idiotismo, imbecilidad, retrasos mentales.

El discípulo más importante de Giné y Partagás es el ya citado Galcerán Granés. Este autor lleva la actitud positivista de Giné a posturas mucho más radicales. Un buen ejemplo de su pensamiento lo encontramos en sus comentarios a la traducción al castellano del Tratado de Psiquiatría del autor americano Hammond ${ }^{41}$. Critica la clasificación de este autor, cercana al modelo de Esquirol, por su falta de carácter científico y se hace defensor del uso en psiquiatría de los métodos en boga en otras ramas de la medicina, que llegaba a su apogeo con el comienzo de la aplicación sistemática del método experimental. Es ilustrativo de esta actitud un trabajo de Galcerán Granés, donde resume la evolución histórica de la psiquiatría ${ }^{42}$. Recuerda en primer lugar la filosofía antigua, que consideraba a la locura como un ente ajeno al organismo y originado por la influencia de los espíritus que regulaban el psiquismo. A esta

40 Plumed, J.; Rey, A. (2004), «Las historias clínicas del Manicomio Nueva Belén», Frenia, IV, 77-99.

41 Hammond fue un prestigioso frenópata norteamericano, cuyo tratado de psiquiatría fue traducido en nuestro país. Ver: HAMMOND, W. A. (1888). Tratado práctico de la locura en sus relaciones médicas, Madrid, Imp. Nicolás Moya.

42 GALCERÁn GrANÉS, A. (1880), «De la naturaleza de la locura», Independencia Médica, 12 (16), pp. 393-395. 
le siguió la medicina griega, que trajo una psicología dominada por la metafísica. Sin embargo, la teoría humoral propugnada por Hipócrates supone para Galcerán Granés el primer acercamiento hacia la vesania como una enfermedad del cuerpo. Cree el autor que este primer paso se frena con la aparición del catolicismo y la filosofía escolástica, que ligan la locura a la idea de pecado.

Para Galcerán Granés la nueva medicina empezó con Pinel, que es el primer psiquiatra que concede a la locura la categoría de enfermedad y define como método de la ciencia la observación. El siguiente paso fue la unión de la psicología y la fisiología en el estudio de la enfermedad mental. Los autores que iniciaron este proceso empezaron a conocer los órganos donde se gesta la enfermedad y las relaciones funcionales que ligan a unos elementos con otros. Sin embargo, no podían eliminar la idea clásica del valor de la subjetividad, un lastre para la mentalidad de Galcerán. Así, por último, se llega al máximo desarrollo de la psiquiatría con la aplicación a este campo del método experimental. En este estadio, puede definir la razón como: «la integridad y normal reciprocidad de las funciones cerebrales entre sí y entre los agentes internos y externos que con el cerebro tienen correspondencia». Estas ideas marcan los principios ideológicos de la escuela positivista en psiquiatría, sostenida por las teorías positivistas de Comte, del que toma en el artículo su hipótesis sobre los tres estadios en el desarrollo del intelecto: el teológico, metafísico y positivo ${ }^{43}$.

El intento de encontrar una clasificación que se ajuste a estos principios es una constante en Galcerán Granés, el autor del siglo XIX que más esfuerzo dedicó al problema de la clasificación en psiquiatría. Su primera gran clasificación recibe el título de: «Responsabilidad civil de los enajenados». Como el nombre indica, el problema de la psiquiatría forense se convierte en la justificación del trabajo. Este fue leído por su autor en el Congreso Frenopático del Manicomio Nueva Belén de $1883^{44}$ y publicado íntegramente el año siguiente

43 Augusto Comte es el fundador de la filosofía positivista. En el Diccionario de Ciencias Eclesiásticas encontramos la siguiente definición: «filósofo francés considerado como fundador de la filosofía positivista»«Considera todas las religiones como una ficción y niega todas las verdades metafísicas, diciendo que esta es una ciencia quimérica»«El método para llegar al conocimiento de la verdad es observar y clasificar los hechos particulares, sacando por inducción las leyes que presiden y determinan la existencia de los fenómenos sensibles, y nada es verdadero sino porque está garantizado por la experiencia y la inducción, que es la verdadera ciencia positiva. Claro está que el sistema de Comte conduce derechamente al materialismo». En: V.V.A.A (1885), Diccionario de ciencias eclesiásticas. Valencia, Imp. Domenech, Tomo III, pp. 90-91.

${ }_{44}$ Sobre el desarrollo y contenido de este congreso se puede consultar: VILLASANTE, O. (1997),»Primer Certamen Frenopático Español (1883). Estructura asistencial y aspectos administrativos», Asclepio, 49 (1), pp, 79-93. 
en la Gaceta Médica Catalana ${ }^{45}$. Toda la clasificación gira en torno a la voluntad humana y en qué manera está condicionada en las diferentes patologías. Para Galcerán Granés, la voluntad era la facultad psíquica más importante, de modo que era primordial definir, a partir de ella, los fenómenos psíquicos.

Ya hemos comentado la importancia que, para la psiquiatría del siglo XIX tenía esta facultad. Si hasta ahora el libre albedrío era defendido por los autores espiritualistas como consecuencia de la independencia del alma, ahora Galcerán propone un modelo unitario y mecanicista, basado en la físiología nerviosa ${ }^{46}$.

La mentalidad fisiopatológica y la introducción de la física y la química como parte integrante del saber médico ${ }^{47}$ son la base de la clasificación que estudiamos. Está muy influida por los planteamientos del psicólogo francés Ribot, que introdujo en Francia las ideas del funcionalismo dinámico y los principios del evolucionismo de Jackson y tuvo una influencia clave en la psiquiatría catalana. Su idea de la voluntad como una facultad superior que se localiza en la corteza cerebral, la zona evolutivamente más tardía, también fue asimilada por Galcerán Granés.

Partiendo de la base de que sólo podemos entender la voluntad como una facultad integrada con las otras, Galcerán Granés propone una clasificación de la enfermedad mental en base a los siguientes elementos: en primer lugar, los actos según sea su origen, cerebral o medular. Después, las motivaciones de dichos actos, que considera el autor la esencia de la locura. De hecho, piensa que los locos tienen plena conciencia de sus actos y de su valor moral; «son

45 GAlCERÁn GRANÉS, A. (1884a) «Responsabilidad parcial de los enajenados. Datos prácticos para conocerla y graduarla», Gaceta Médica Catalana, 7, 580-90; 621-29; 648-57; 689-99; 714-22; 745-52.

46 En una conferencia dada en Nueva Belen, Galcerán Granés dice: «el libre albedrío es una ilusión, y la causa de esa ilusión es la inconsciencia que tenemos, en muchos casos, de los motivos inmediatos de una determinada resolución. Lo que sustituye al libre albedrío en el terreno de la actual ciencia es la volición.»«Con esto queda demostrado, que en vez de una entidad, de una fuerza material que dirija nuestras acciones con conciencia innata, además, de lo bueno y de lo malo que aquellas puedan realizar, es la volición un proceso puramente orgánico, mejor, un proceso cerebral, y como tal, un producto de correlación e integración de fuerzas materiales». Es un ejemplo claro del pensamiento ateo y positivista del autor. Ver. GALCERÁN GRANÉS, A. (1884b) «El determinismo en la voluntad y el libre albedrío: crítica de ambas teorías», Revista frenopática Barcelonesa. 3, p. 369.

47 Para LÓPEZ PIÑERo (2002), p. 517, la orientación fisiopatológica de la medicina entendió los trastornos funcionales como procesos energéticos o materiales, investigándolos respectivamente con los recursos de la física y la química. 
locos en cuanto a la valoración de los motivos que los han determinado» ${ }^{48}$. Este fundamento psicológico le lleva a formular una ley: la responsabilidad de las acciones humanas está en razón directa de la libertad de volición e inversa de la intensidad de los motivos ${ }^{49}$. Todo el proceso depende de la cantidad de energía de que dispone cada estructura cerebral que condiciona el proceso.

Hechos como el descubrimiento de Hemholz sobre la conducción eléctrica en el tejido nervioso y la ley de Fechner para medir la percepción de la intensidad de un estímulo creaban la ilusión de encontrar una psiquiatría científica que pudiera regirse con la misma fidelidad a un canon objetivo como ocurre con la química o con las matemáticas.

Los parámetros que hemos comentado permiten clasificar toda la patología mental. Como ejemplo, veamos la concepción del autor de la demencia. Se considera como un trastorno general por defecto de la voluntad. Hay una descripción clínica del proceso en el tiempo: las emociones son menos vivas, la afectividad se extingue lenta y gradualmente; la indiferencia la sustituye y la apatía se impone. El individuo tiene conciencia de este cambio progresivo y aunque no se lo explica le aflige profundamente. Sus lágrimas son fáciles y frecuentes, se angustia y enoja por cualquier cosa. Al avanzar la demencia, el grado de debilidad de la memoria es tan elevado que el enfermo olvida sus palabras y sus acciones inmediatamente después de cometidas. Hay también una parálisis de la sensibilidad, el movimiento y la voluntad.

La debilidad de la memoria se debe a que la fuerza de las células cerebrales se integra más difícilmente con los agentes internos o externos. El movimiento molecular nutritivo es deficiente, y por tanto la conservación de las impresiones y su poder de reproducción son pobres. La distracción y la inatención son un corolario de la debilidad de la memoria. Estos factores reunidos determinan una asociación incompleta o poco potente. Las operaciones mentales no pueden ser elevadas o abstractas y sí concretas y automáticas ${ }^{50}$.

48 GALCERÁN GRANÉS (1884a), p. 586.

49 Esta idea es análoga a la hipótesis, antes comentada, de la influencia de las pasiones como causa de la pérdida de la razón. Así, cuando habla de las alteraciones de la voluntad por exceso, parcial y consciente escribe: «El representante fisiológico es la pasión. Cuando las corrientes endoperiféricas propias de las pasiones llegan al cerebro, el individuo lucha, procura distraerse, pero al final cede». «En estos enfermos, la sensibilidad orgánica, transmitiendo potentes corrientes, y el exagerado reflejismo cerebral, convirtiendo estas corrientes en la voluntad fatal, es lo único enfermo». GALCERÁN GRANÉS, A (1884a), p. 626.

50 GALCERÁN GRANÉS, A. (1884a), p. 697. 
De todos modos, la clasificación más importante y citada de Galcerán Granés fue el «Ensayo de clasificación anatomopatológico de las vesanias» ${ }^{51}$. Comienza el texto hablando de la evolución que ha llevado la clasificación en psiquiatría. En primer lugar para este autor, la nosología era puramente empírica, basada en el síntoma; después empezaron los frenópatas a basarse en la lesión anatómica y, por último, en la etiología. Es decir, la nueva psiquiatría se había basado en el modelo hipocrático del síntoma hasta llegar a los nuevos autores, en los que destaca a Luys ${ }^{52}$. La clasificación de Galcerán Granés intenta ser una integración de las tres perspectivas. Parte del principio de que todo síntoma se produce por: exageración, disminución o supresión del funcionalismo cerebral, con lo que las enfermedades se pueden clasificar por: su carácter irritativo, depresivo o destructivo. Establece una división funcional y anatómica del cerebro: lóbulo frontal (intelecto-volitivo) y parieto-temporooccipital (sensitivo-sensorial), de modo que cada enfermedad mental supone la afectación de uno u otro lado. A su vez, las enfermedades son localizadas o consecuencia de una lesión difusa, como en la idiocia o la demencia. Se considera que la enfermedad mental es de tipo estático si se produce por un defecto anatómico visible como en la parálisis general y dinámica si se debe a una alteración funcional, básicamente el riego vascular.

Así, los supuestos descubrimientos biológicos hacen que podamos hablar de enfermedades mentales y no de síndromes. Además, la clínica es explicable con criterios anatómicos o fisiopatológicos. Con ello, se produce el salto de la observación clínica defendida por Pinel y Esquirol, de un contínuo en el que se podían distinguir géneros o especies, al estudio de enfermedades concretas y objetivables que se ajustan al método científico.

Si esto fue cierto en el caso de la parálisis general, en los demás trastornos fue algo puramente especulativo y racionalista, movido por la necesidad de defender la psiquiatría como una disciplina científica equiparable a las demás ramas de la medicina y que, por tanto, se beneficiaría de los avances médicos conocidos en la medicina clínica.

Como ejemplo, en su clasificación define las ideofrenias como: la exaltación maníaca, la manía general aguda y la melancolía delirante. Para este autor, se trata de una lesión frontal por hiperemia, en la cual hay un trastorno

51 GALCERÁN GRANÉS, A. (1889), «Ensayo de clasificación anatomopatológico de las vesanias», Siglo Médico, 36, 407-9; 419-22; 436-8; 454-7; 470-2; 486-8.

52 Luys fue un autor francés de orientación organicista que fue abundantemente citado en la prensa médica española de la época. Fue traducido: LUYS, J. (1891), Tratado clínico y práctico de las enfermedades mentales, Madrid, Imp. Enrique Teodoro. 
evidente del juicio y la asociación de ideas. Supone como sustrato anatómico una lesión funcional irritativo-hiperémica, siguiendo a Luys, que afecta a vasos y células causando una lesión microscópica: la degeneración gránulograsoso-pigmentaria.

Las nuevas enfermedades mentales, como la locura sistematizada progresiva, también tienen un lugar en la clasificación. Escribe que, aunque la anatomía patológica no es definitiva, dado que al principio predominan las alucinaciones que originan el delirio, cabe suponer que la dolencia asiente en los lóbulos esfenoidales y occipitales. Es decir, hay una actitud empirista, según la cual sólo los datos observables son válidos para establecer una clasificación y se acepta que toda manifestación mental se correlaciona con una alteración anatómica o funcional. Sin embargo, el trabajo está basado en hipótesis especulativas. Así, por ejemplo, en la medida en que se relacionan los trastornos visuales y auditivos con los lóbulos occipitales y esfenoidales, cabría relacionar una alucinación con estas zonas.

Todo el proceso evolutivo de la enfermedad se describe como un proceso fisiológico bien determinado: «en el comienzo del mal, se encuentra el enfermo en estado permanente de eretismo psicosensorial, estado que, creciendo progresivamente de intensidad, determina un acceso de delirio general agudo con fuerte reacción mórbida y un aumento de la temperatura general y craneana, al tiempo que se produce una desasimilación exagerada de sustancias orgánicas, sobre todo nerviosas».»Andando el tiempo, pierde su enfermo su potencia mental hasta llegar a la demencia, significando que el proceso ataca cada vez más profundamente la sustancia cortical, destruyéndola» ${ }^{53}$.

Este proceso de análisis se extiende en el trabajo a todos los trastornos mentales conocidos.

Sin embargo, años más tarde, en la recopilación de sus lecciones de psiquiatría $^{54}$ reconoce que el estadio final de desarrollo de la medicina mental estaba lejos de llegar: «las aspiraciones de la actual ciencia no son otras que las de fundar una clasificación etiológica para todas las enfermedades; pero son aún de tal suerte las dificultades y tan limitadas las conquistas conseguidas, que hasta ahora es totalmente imposible llevarlas a cabo de una manera exclusiva». «Son muchos más los inconvenientes que ofrecen las clasificaciones exclusivamente fundadas en el carácter sintomático o anatomopatológico, pues sobre no permitir sino bajo el punto de vista sintético, la aprecia-

53 Galcerán GranÉs, A. (1889), p. 437.

54 GAlCERÁn GRANÉS, A. (1895), Neuropatología y psiquiatría generales, Barcelona, Imp. Casa Provincial de la Caridad. 
ción de una sola contingencia de la enfermedad, supone esta un concepto relativamente insignificante en comparación con el que implica la condición de causa». Al no tener un conocimiento claro del factor causal, «el concepto anatomopatológico debe, todavía en la época presente»«servir de fundamento de clasificación de las enfermedades que al sistema nervioso afectan $»^{55}$. No es extraño su progresivo interés por las ideas degeneracionistas ${ }^{56}$, así como el esfuerzo constante en sus lecciones de buscar relaciones firmes entre los conocimientos neurobiológicos, los fenómenos clínicos y las teorías psicológicas asociacionistas y basadas en la teoría de facultades.

De hecho, la crítica presente en otros autores ya vistos sobre la falta de veracidad de las supuestas lesiones y trastornos funcionales está ausente en Galcerán Granés, que define un cuerpo de conocimiento nuevo y moderno que lo legitiman a él y a su escuela como la vanguardia científica de la nueva especialidad.

Si aún Giné y Partagás defendía el concepto de alma a través de la idea de voluntad, para Galcerán Granés todo fenómeno mental es consecuencia de alteraciones de la función orgánica: «donde quiera que la corriente nerviosa se detiene, que la célula está tensiva, allí empieza la voluntad. Desde el instante que el movimiento molecular tensivo que caracteriza la atención se convierte en corriente, cúmplese fatalmente la voluntad ${ }^{57}$. El concepto de moralidad tradicional lo reformula de acuerdo con principios biológicos ligados al evolucionismo y al cientifismo moderno: «las acciones son moralmente buenas cuando tienden al fin de la conservación del individuo, de la especie o de la sociedad $»^{58}$.

Fig. 3. CLASIFICACIÓN ANATÓMICA DE LAS VESANIAS DE GALCERÁN GRANÉS.

1. Clase 1: Periencefalitis localizada.

1.1. Subclase: frontales.

1.1.1. Géneros: Perimeningoencefalitis frontal proliferativa degenerativa.

1.1.1.1. Especie: Parálisis general progresiva.

1.1.1.2. Especie: Ideofrenia.

1.2. Subclase: frontoparietales.

55 GALCERÁN GRANÉS, A. (1895), pp. 238-239.

56 Ver: PlumeD, J.; REY, A. (2001) «La introducción de las ideas degeneracionistas en la España del siglo XIX», Frenia, II, (1), 31-48, pp. 45-46.

57 GALCERÁN GRANÉS, A. (1895), p. 122.

58 GALCERÁN GRANÉS, A. (1895), p. 141. 


\subsubsection{Género: perimeningoencefalitis frontoparietal crónica exudati-} va.

1.2.1.1. Especie: locura impulsiva.

1.3. Subclase: parietooccipitales.

1.3.1.1. Género: perimeningoncefalitis parietooccipital hiperémica. 1.3.1.1.1. Locura sensitiva.

1.4. Subclase: témporooccipitales.

1.4.1.1. Género: perimeningoencefalitistemporooccipital crónica.

1.4.1.1.1. Especie: locura sensorial.

1.4.1.2. Género: perimeningoencefalitis esfenoidal evolutiva.

1.4.1.2.1. Especie: locura sistematizada progresiva.

1.4.1.3. Género: perimeningoencefalitis esfenobasilar degenerativa.

1.4.1.3.1. Especie: demencia melancólica.

2. Clase 2: Periencefalitis difusa.

2.1. Subclase: Vasculares.

2.1.1. Géneros: isquémicas, neuroparalíticas e inhibitorias.

2.1.1.1. Especies: locuras epiestesiopáticas (hiperestesia retiniana, timpánica o cerebroespinal).

2.2. Subclase: parenquimatosas (agudas).

2.2.1. Géneros: arteriosclerosis generalizada, diatésicas, hiperémicas, infectivas, generativas.

2.2.1.1. Especies: locura por ateroma, reumática, gotosa, sifilítica, tuberculosa, tífica, delirio agudo vesánico, locura pelagrosa.

2.3. Subclase: intersticiales (crónicas).

2.3.1. Género: tóxicas (perimeningoencefalitis intersticial proliferativa); escleróticas (perimeningoencefalitis difusa atrófica).

2.3.1.1. Especies: locura saturnina, locura alcohólica, locura hidrargírica, locura por el sulfuro de carbono, locura nicotínica, locura arsenical, locura morfínica.

2.4. Subclase: Totius substantiae.

2.4.1. Género: meningoencefalitis regresiva atrófica, meningoencefalitis destructiva.

2.4.1.1. Especie: Demencia terminal.

3. Clase: Agenesias cerebrales.

3.1.1.1. Especies: imbecilidad, idiotismo, cretinismo, aztequismo, locuras degenerativas (circular-doble forma-periódica). 
LOS PRIMEROS INTENTOS DE CLASIFICACIÓN BASADOS EN LA TEORÍA DE LA DEGENERACIÓN

Otros autores han comentado el escaso apoyo presente en la literatura médica española de la última parte del siglo XIX a la doctrina de la degeneración $^{59}$. Sin embargo, algunos frenópatas fueron acercándose a estas ideas en cuanto esta teoría proponía una clasificación etiológica y una explicación supuestamente científica de la enfermedad mental. De hecho, se tradujeron abundantes artículos del máximo representante de la escuela, Valentín Magnan $^{60}$, donde se abordan importantes cuestiones nosológicas. Así, en un artículo traducido en el Siglo Médico ${ }^{61}$ hace una crítica a Morel por introducir supuestas entidades distintas, como el delirio emotivo ${ }^{62}$ o la locura hipocondríaca $^{63}$, cuando el criterio de clasificación, antes que en diferencias clínicas, debe residir en el fondo patológico común: el desequilibrio del degenerado ${ }^{64}$.

Hubo algunos amagos de clasificación con estos criterios en la psiquiatría española. Ots Esquerdo, uno de los autores más prolíficos, fue un ferviente

59 Ver: CAMPOS, R. (1998), «Teoría de la degeneración y medicina social en España», Llull, 21, 333-356.

60 Ver: REY, A. (1981) La introducción del moderno saber psiquiátrico en la España del siglo XIX, Tesis doctoral, Valencia, Tomo I, p. 63. 104-6.

61 Magnan, V. (1887), «Consideraciones generales sobre la locura», Siglo Médico, 34,

62 Para Morel, el delirio emotivo no era una forma de locura sino una neurosis, una enfermedad de las emociones. Correspondía a un tipo de idea fija y acto anormal cuya existencia no suponía una afectación de las facultades intelectuales. BERRIOS, G.E. (1995), Obssesive Compulsive Disorder. En: Berrios G.E.; PorTer, R., A History of Clinical Psychiatry, Londres, Athlone, pp. 576-577.

63 La clasificación de enfermedades mentales de Morel (1860) se dividía en 6 grupos: degeneración hereditaria; degeneración por intoxicación; degeneración determinada por la transformación de ciertas neurosis: histérica, epiléptica e hipocondríaca; degeneración idiopática; locuras simpáticas y demencia. En: HuERTAS, R. (1992), «Madness and Degeneration. From «fallen angel» to mentally ill», History of Psychiatry, iii, 391-411, p. 404.

64 Para Magnan, el desequilibrio es el mecanismo fisiopatológico fundamental en el proceso de la degeneración. «Puede resolverse en los siguientes términos: destrucción o interrupción del desarrollo de ciertas partes; hiperactividad momentánea o permanente de otras; o disminución momentánea y duradera de la actividad de otras». «Las consecuencias inmediatas de este desarrollo son: la supresión de ciertas funciones; el automatismo de centros hiperexcitados y la inhibición exagerada sobre los centros más válidos. Toda esta serie de fenómenos se combinan, se suceden, de forma que la medicina asiste a una verdadera ataxia cerebroespinal». En: Magnan, V.; Legrain, M. (1895). Les dégénérés, Paris, Masson, pp. 100-102. 
degeneracionista. Respecto a la nosología vigente en el momento la considera como insuficiente «a pesar de los grandes avances en la patogenia y la clasificación de la enfermedad mental» ${ }^{65}$. Esto le lleva a enunciar una clasificación propia ${ }^{66}$. Basa su clasificación en dos grupos: aquellos trastornos cuyo único origen está en la afectación degenerativa hereditaria y aquellos en que la disfunción procede de una causa externa, ya sea por tóxicos o por enfermedades orgánicas conocidas. De hecho, Ots Esquerdo prácticamente copia el esquema nosológico de Magnan modificando dos elementos: incluye las formas neurósicas $^{67}$ en el primer grupo, que Magnan diferenció y desdobla el grupo de estados degenerativos en las formas pronunciadas y exageradas. Con ello, enfatiza la idea de la degeneración como el mecanismo fisiopatológico universal en la enfermedad mental, de modo que los trastornos supuestamente causados por lesiones reconocibles en el cerebro no son sino otras formas degenerativas. Ello acentúa la falta de límites entre enfermedad neurológica y psiquiátrica, en un momento en que la nueva neurología estaba viviendo un momento claro de expansión ${ }^{68}$. La diferencia entre las diversas enfermedades mentales no reside en la lesión específica que define a cada una, sino en el grado en que el proceso degenerativo ha llegado a producirse. Con ello, la nosología psiquiátrica supuestamente llegaba al punto máximo de su evolución: basarse en la etiología y la fisiopatología.

Este planteamiento de la degeneración como el proceso que explica todas las formas de locura no fue compartido por la totalidad de los frenópatas de orientación degeneracionista. Mateo Bonafonte, al hablar de la clasificación de la enfermedad mental, defiende los postulados del psiquiatra francés Regis $^{69}$. Este autor divide la patología mental en dos grupos: en primer lugar, los no degenerados: «en ellos, los varios tipos de vesanias, manía, melancolía,

65 Ots Esquerdo, V. (1893), «Locura alcohólica», Siglo Médico, 40, 470-2.

66 Ots Esquerdo, V. (1894), «Nueva clasificación frenopática», Revista Médica de Cirugía Práctica, 34, 51-3.

${ }^{67}$ En el siglo XIX, se entiende como neurosis aquellos trastornos mentales con lesión cerebral reconocible. Ver sobre el tema: GARRABÉ, J. (1996), La noche oscura del ser, México, Fondo de Cultura Económica, p. 33.

68 Ver: HirschmülleR, A. (1999), «The Development of Psychiatry and Neurology in the $19^{\text {th }}$ century» History of psychiatry, 10, 395-423.

69 Regis fue uno de los autores más referenciados por los psiquiatras españoles en el primer tercio del siglo XX. Escribió en varias ediciones su Prècis de Psychiatrie, que fue el manual más importante de la psiquiatría francesa del período y uno de los escasos tratados de psiquiatría traducidos en España. Ver: Postel, J.; QueTel, C. (1983), Historia de la psiquiatría. México, Fondo de Cultura Económica, p. 730. 
locuras parciales sistematizadas, etc, prescindiendo de su origen y condiciones de herencia ya indicadas, se realiza la marcha del proceso con una pureza de síntomas que basta haberle leído una vez para que no exista duda, la sistematización es perfecta, y el megalómano no degenerado se identifica perfectamente con su papel y la transformación de su personalidad es completa» ${ }^{70}$. Después, tenemos el grupo de enfermos afectados por el proceso degenerativo: «en los degenerados, no existe tal sistematización, el megalómano rey o príncipe nos confiesa que es un jornalero que gana dos pesetas, pasa con facilidad del trastorno tipo manía al de melancolía y mezcla abigarradamente las ideas de persecución con las eróticas $\rangle^{71}$. Con esto, vemos que la degeneración deja de convertirse en el mecanismo único de producción de la enfermedad mental, que era una salida teórica extraordinariamente útil para salir de las graves dificultades que presentaba aplicar la medicina moderna al campo de la psiquiatría. Así, queda delimitada al campo de los trastornos más complejos y que resultan más difíciles de encasillar.

Martínez Valverde señala los cuatro tipos posibles de clasificación en psiquiatría: las psicológicas, cuyo fundamento son los desórdenes psíquicos; las sintomáticas, cuyo fundamento son los síntomas; las etiológicas y las anatomopatológicas. «Como se comprende a primera vista, las divisiones que aceptan el criterio sintomático y psicológico son defectuosas». "Las clasificaciones etiológicas y anatómicas son las más científicas». «El criterio anatomopatológico se halla con dificultades por ahora insuperables, porque son pocas las lesiones bien conocidas y determinadas». En este sentido, la doctrina de la degeneración es la única que permite una clasificación etiológica, como sucede en la medicina interna con la patología infecciosa: «Morel fue el primero que comprendió el valor de tal base e intentó, fundado en ella, formular una clasificación causal, y desde entonces la inmensa mayoría de los autores le han imitado, siguiendo sus huellas» ${ }^{72}$.

Este autor asume la diferencia entre síndrome y enfermedad: «hay en frenopatología varios estados, conjunto de síntomas, cuadros sindrómicos más o menos perfectamente definidos, que por muchos, casi por la mayoría, han sido estudiados como si fueran entidades nosológicas independientes». Partiendo de esta base defiende la clasificación de Regis, distinguiendo la locura como accidente desgraciado en la vida de un enfermo previamente sano o la

70 Bonafonte, M. (1900), Degeneración y locura, Zaragoza, Imp. Manuel Ventura, p. 30.

71 BOAFONTE, M. (1900), p. 42.

72 Martínez y VAlVERDE, J. (1900), Guía del diagnóstico de las enfermedades mentales, Barcelona, José Espasa, pp. 99-100. 
consecuente al proceso degenerativo. Respecto a este último mecanismo, dice en su libro: «estos trastornos, verdaderos vicios de organización psíquica, son expresión de anomalías orgánicas, comúnmente hereditarias». Al menos, hay un proceso neurofisiológico determinado que se conoce como causante de los cuadros psiquiátricos más atípicos.

FIG. 4. ClASIFICACIÓN DE OTS ESQUERDO.

1. Degenerados hereditarios.

1.1. Formas atenuadas.

1.1.1. Simples (psicopáticas y neuróticas).

1.1.2. Complejas.

1.1.2.1. De curso típico: locura circular.

1.1.2.2. De curso atípico: megalomaníaca, persecutiva y comunicada.

\subsubsection{Pronunciadas.}

1.1.3.1. Regulares: locuras impulsivas y obsediantes; delirios poli y monomorfos; delirios tóxicos.

1.1.3.2. Exageradas: individuales (imbecilidad e idiotismo) y cósmicas (cretinismo y astequismo).

2. Degeneración debida a causas externas, enfermedades orgánicas o tóxicos.

2.1. Comunes: parálisis general, delirio agudo y demencia senil u orgánica.

2.2. Especiales: específicas (pelagra, sífilis, reumatismo cerebral) o no específicas (tumor, reblandecimiento y hemorragia).

\section{LA CLASIFICACIÓN DE LA LOCURA EN LA ASISTENCIA MANICOMIAL}

Todas estas sofisticaciones diagnósticas se movían dentro del campo de lo teórico. Sin embargo, la aplicación en la clínica era muy limitada.

En una nota sin autor aparecida en el Siglo Médico $^{73}$ se indican los diagnósticos de los enfermos que ingresaron en el Hospital General de la Villa de Madrid el mes de Mayo de 1855. Tres hombres padecían demencia, cuatro

73 Sin AUtOR (1855), «Nota estadística de los dementes de ambos sexos que durante el mes de Junio ha habido en el hospital general de esta Corte», Siglo Médico, 2, 215. 
fueron diagnosticados de idiotismo y dos de manía. En lo que concierne a las mujeres, dos padecían alucinaciones, tres manía y una demencia. González Navas ${ }^{74}$, director del Manicomio de Toledo, comenta los pacientes y diagnósticos de los enfermos en los años 1858 y 1859 . En el primer año, dos enfermos padecen delirio agudo y sin fiebre; dos son diagnosticados de alucinaciones o ilusiones; trece enfermos reciben el diagnóstico de manía aguda con o sin delirio; catorce de monomanías de todo tipo; tres de melancolía; dos de demencia paralítica y uno de senil. Ya en 1859, de 58 internos, uno padece delirio agudo febril, dieciocho padecen «manías de todo tipo»; tres padecen alucinaciones; treinta y dos están diagnosticados de monomanías; dos sufren de ilusiones; nueve de demencia y tres de imbecilidad.

Es decir, los esquemas diagnósticos son muy simples: no hay una distinción entre la alucinación y la ilusión como enfermedad o como síntoma y se nombran las monomanías o manías sin matices, como corresponde a un régimen de internado en que los aspectos terapéuticos están de lado. En este sentido, Espinosa recoge en su libro la opinión de Ford sobre este manicomio:»no hace ningún honor a la ciencia ni a la humanidad. La locura parece haber alterado al mismo tiempo el intelecto de los enfermos y haber oscurecido el de los que les atienden». «Los internados están amontonados conjuntamente (el monomaníaco, el loco, la histérica) en una confusión miserable o encadenados como bestias salvajes y tratados incluso peor que los criminales, porque las pasiones de los más furiosos son enfurecidas por el trato brutal $\rangle^{75}$.

Lorenzo Livianos y Magraner señalan en su libro sobre las historias clínicas de Perales ${ }^{76}$ la importancia que para este frenópata tuvo el diagnóstico y tratamiento de los enfermos mentales. Recuerdan los autores que Perales fue el artífice de los cambios asistenciales en el Hospital General de Valencia. Este interés se refleja en los diagnósticos. Junto con los grandes trastornos heredados de la nosología de Esquirol, en el diagnóstico de los casos suele señalar aspectos clínicos que considera relevantes para el tratamiento y hace especial hincapié en el curso de la enfermedad; si es aguda, crónica o evoluciona por fases. Así, en un caso de monomanía se señala el carácter de gran-

74 GonZÁlez Navas, Z. (1858), «Consideraciones acerca del hospital de enajenados de Toledo», Siglo Médico, 5, 105-6; 113-4; 121-2; 145-6; 153-4, p. 113.

75 FORD, R. (1845), Handbook for Travellers in Spain, Londres. En: EsPINOSA, J. (1966) La asistencia psiquiátrica en la España del siglo XIX, Valencia, Cátedra de Historia de la Medicina, p. 53.

76 Livianos, L.; Magraner, A. (1991), Historias clínicas psiquiátricas del siglo XIX, Valencia, Ayuntamiento de Valencia, pp. 64-65. 
deza $^{77}$, probablemente por el carácter ominoso para el curso de la enfermedad que se creía en la época que este tipo de delirio tenía. Otro caso está diagnosticado de monomanía continua y crónica: «ni el sillón de fuerza, ni la camisa de igual nombre, ni la dieta prolongada, ni consuelos morales, ni oposición constante a sus ideas, ni conveniencia tácita o clara con ellas, baños de sorpresa, ducha según el método de Leuret, nada en fin ha bastado a conseguir el menor alivio» ${ }^{78}$. En la asistencia manicomial la evolución en el tiempo y la respuesta a los tratamientos es, quizás, el aspecto clave. No es de extrañar que un hombre tan preocupado por el tratamiento de los enfermos mentales consignara de forma casi sistemática este tipo de datos en las historias clínicas.

En los casos clínicos del Manicomio Nueva Belén, un manicomio privado de élite, vemos que los frenópatas de la escuela catalana utilizan, de la misma forma que Perales, las grandes categorías diagnósticas: manía, melancolía, monomanía y demencia. Hay un interés especial en considerar los aspectos relacionados con el curso evolutivo del paciente y se resaltan aspectos clínicos concretos que tienen que ver con la terapéutica. Este interés por la cronología hace que autores como Rodríguez Morini ${ }^{79}$ hablen de diagnósticos como la locura sistemática progresiva y el delirio crónico de evolución sistemática, basados en la patocronia.

Por otro lado, hemos estudiado ${ }^{80}$ cómo en esta institución los tratamientos se centran en los grandes aspectos sindrómicos y no en los detalles diagnósticos. Además, resulta evidente la ausencia de interés por aquellos aspectos teóricos y nosológicos que hemos visto anteriormente en autores como Galcerán Granés. Sólo en determinados casos se introducen complejas justificaciones fisiopatológicas para explicar la utilidad de tratamientos que también se utilizaban en otros centros de forma generalizada.

En uno de los debates más intensos en la época, cómo debía ser el manicomio modelo, se discutía la importancia que la clasificación psiquiátrica debía tener en la estructura de un centro ideal. Así, González Navas ${ }^{81}$ dice que, en un manicomio, los enfermos deberían estar divididos en salas de curables y salas de incurables, condiciones que estaban lejos de darse en la insti-

77 Livianos, L.; Magraner, A. (1991), p. 114.

78 LiVianos, L.; MAgRANER, A. (1991), p. 110.

79 RodríGuez Morini, A. (1885), «Delirio crónico de grandezas (locura parcial)», Revista_Frenopática Barcelonesa, 5, 39-47.

80 Plumed, J.; ReY, A. (2004).

81 GonZÁleZ NAVAS, Z.(1858), pp. 121-122. 
tución que regentaba. Su sucesor en el Manicomio de Toledo, Sánchez ${ }^{82}$, comenta las pésimas condiciones en que recibe la dirección del centro: falta de enfermería, condiciones deficientes de higiene, falta de ropa de abrigo... Destaca que no existe una distribución de enfermos por patologías, como sugieren la mayoría de los autores. En referencia a Pinel y sus discípulos escribe: «al estudiar los manicomios se fijaron, como una de las partes principales, en la distribución de las secciones y en la conveniencia de separar a los enajenados según su forma de locura. Comprendieron los inconvenientes que resultarían si se hallasen los afectos de una misma categoría de perturbaciones mentales, e hicieron su separación con arreglo a sus clasificaciones o a su manera de pensar en este punto ${ }^{83}$. Simarro ${ }^{84}$ escribe que un manicomio ha de organizar a los enfermos de acuerdo con su diagnóstico, especialmente en los centros de agudos. Por otro lado, en los centros de crónicos debe predominar la actividad en común de los enfermos, como actividad rehabilitadora. También destaca la importancia que deberían tener las unidades creadas para tratar patologías especiales, como imbecilismo, cretinismo, morfinomanía y alcoholismo. Sin embargo, el hecho es que en su etapa asistencial como director del manicomio de Leganés no encontró ni pudo implantar estas condiciones y en un estudio se evidenció que los diagnósticos de sus enfermos se ajustaban, básicamente, a la nosografía de Esquirol ${ }^{85}$. Por otra parte, en un trabajo reciente se demuestra que, durante la segunda mitad del siglo XIX, los diagnósticos psiquiátricos presentes en las historias clínicas de Leganés no sólo pertenecen a la nosología de Pinel y Esquirol, sino que también incorporan otros diagnósticos procedentes de la escuela francesa, como la parálisis general, la demencia precoz o la locura de doble forma. Además, a finales de siglo se fueron adoptando las nuevas categorías diagnósticas procedentes de la psiquiatría alemana. Los autores señalan la contradicción entre este aparente virtuosismo diagnóstico y la pobreza de tratamientos, así como las deficientes condiciones higiénicas del centro, traducidas en una alta mortalidad ${ }^{86}$.

82 SÁnchez, F. (1877), «Memoria y datos estadísticos sobre el «nuncio de Toledo», Anfiteatro Anatómico Español, 5, 209-210; 223-224; 235; 248; 258-9; 273-5.

83 SÁNCHEZ, F. (1877), p. 235.

84 Simarro, L. (1893), «Proyecto de un manicomio», Siglo Médico, 40, 789-91; 809-11.

85 Ver: Moro, A.; Villasante, O. (2001) «La etapa de Luis Simarro en el manicomio de Leganés», Frenia, I, I, 97-119.

${ }^{86}$ Mollejo, E.; Del CuRA, M.; HuerTAs, R. (2004), La práctica clínica en el Manicomio de Leganés (1852-1936) $1^{a}$ aproximación al estudio de sus historias clínicas» En: MARTíNEZ Pérez, J; Porras Gallo, I., Samblás Tilve, P., Del Cura González, M, La Medicina ante 
A finales de siglo, el tema asistencial más debatido era la conveniencia de ingresar a un enfermo en el manicomio o bien centrar la atención psiquiátrica en el domicilio del paciente. Sanz y Gómez piensa que el criterio básico de decisión es que peligre la vida del paciente y de los que lo rodean. Comenta: «el enajenado curable, en el momento en que su cerebro mejora»«al verse rodeado de otros pensionistas y entre los muros o verjas de un manicomio, sufre tal conmoción que, abulliándose, achicado, amilanado en sus decisiones, tal es su abatimiento que la recaída suele ser la resultante de todos estos procedimientos perjudiciales» ${ }^{87}$. Sin embargo, Pablo Llorach ${ }^{88}$, director del Instituto Frenopático, importante institución privada catalana, piensa que es inútil cualquier tratamiento físico o moral fuera de la institución del manicomio. Es decir, para las instituciones privadas el ingreso temprano era el factor pronóstico más importante para una buena evolución.

Escuder, como Sanz y Gómez, también considera que la única función de la institución manicomial es coercitiva: «los manicomios hoy cumplen en su mayoría la misión casi única de tener encerrados a los locos, aislándoles y separándoles del contacto de sus familias, que ordinariamente les perjudican. Además, el loco suelto por las ciudades perturba, desordena y a veces atenta contra los demás. Fue preciso meterlo donde no pudiera salir y molestar» ${ }^{89}$. En un manicomio eficaz, el tratamiento debería estar basado en el tipo de patología que sufre el enfermo. Al respecto de este tema, escribe: «cada enfermo exige un tratamiento especial, grados de libertad diferente, distinta comida, diversa habitación». «La cama de un epiléptico no debe ser igual a la de un melancólico. El cuarto de un maniaco necesita un revestimiento elástico para que no se estrelle contra la pared. Toda vigilancia es escasa con los suicidas. ¿En qué manicomio español se tiene el lecho para las histeroepilépticas? $\rangle^{90}$ De hecho, las distintas patologías mentales requieren un tratamiento específico en cuanto a las manifestaciones conductuales más groseras, sin que los detalles diagnósticos sean de interés práctico. Aun así, estas necesidades terapéuticas no se satisfacen en la asistencia pública.

el nuevo milenio: una perspectiva histórica, Albacete, Universidad de Castilla la Mancha, pp. 149-162.

87 SANZ Y GómEZ, T. (1893), «Tratamiento de la locura: indicaciones y contraindicaciones de la reclusión manicomial», Siglo Médico, 40, 726-8; 742-5; 758-60; 773-6, p. 759.

88 Llorach, P. (1863), «Necesidad de una clasificación racional de las frenopatías», Siglo Médico, 10, 114-119.

89 EsCuder, J.M. (1895), Locos y anómalos, Madrid, Imp. Rivadeneyra, p. 309.

90 ESCUDER, J.M. (1895), p. 311. 


\section{CONCLUSIONES}

La clasificación psiquiátrica en España sigue, salvo en honrosas excepciones, de forma escasamente original las corrientes ideológicas europeas. Principalmente la francesa, pero también autores como Guislain y Griesinger influyeron en frenópatas tan importantes como Giné y Partagás.

En la primera parte del siglo, la nosología está marcada por la influencia de Pinel y Esquirol, y los autores espiritualistas mantuvieron su influencia hasta la segunda mitad del siglo. Factores como: el desarrollo del cientifismo, el carácter laico de la ciencia de la segunda parte del siglo y el desarrollo de especialidades como la neurología favorecieron las corrientes organicistas en psiquiatría. Factores sociales, como la necesidad de ocupar un puesto de prestigio entre las nuevas especialidades médicas o defender la excelencia de la práctica privada no fueron ajenos a los modelos nosológicos, especialmente de los frenópatas catalanes.

La importancia de la etiología en Medicina y la falta de causas objetivas en los trastornos mentales pudieron favorecer la aparición de clasificaciones de orientación degeneracionista. Algunos frenópatas españoles adoptaron de forma casi idéntica algunas clasificaciones significativas propuestas por la escuela francesa, con lo que dispusieron de un mecanismo patogénico que justificaba el trastorno mental, de acuerdo con la corriente positivista.

Por otro lado, todo este debate teórico estaba ajeno a la práctica asistencial. Esta era precaria en la asistencia pública y escasamente sofisticada en la privada, ajena a las complicaciones nosológicas debatidas en la literatura. Sin embargo, la falta de una organización manicomial basada en las distintas modalidades de enfermedad mental fue ampliamente debatida. 